\title{
Role of Fluorodeoxyglucose - Positron Emission Tomography Scan in Detection of Residual Pus Pocket Post-Operatively in Tuberculosis of Spine and Juxta Articular Tuberculosis
}

\author{
Khandelwal RCS ${ }^{*}$, Sabari Nathan ${ }^{1}$, Tanmay Deo ${ }^{1}$, Jagdish Madhukar Dhake ${ }^{1}$ and Nandini S Shetty ${ }^{2}$ \\ ${ }^{1}$ Department of Orthopaedics, King Edward Memorial Hospital, Mumbai, India \\ ${ }^{2}$ Bone and Joint Clinic, Sion, Mumbai, India
}

\begin{abstract}
The aim of this article is to review the main applications and advantages of Fluorodeoxyglucose PET in imaging of tuberculosis (TB) of spine post-operatively. In TB, granulomas typically demonstrate increased Fluorodeoxyglucose uptake, and areas of active TB can be differentiated from old or inactive disease by dual time point imaging. However, standardized uptake value measurements are high in both TB and malignant lesions, with significant overlap that limits their usefulness. In spinal TB, Fluorodeoxyglucose PET detects more tuberculous lesions than CT, is of value in assessing response to tuberculostatic treatment. Fluorodeoxyglucose PET can also be considered a marker of disease status in patients with HIV and TB co-infection. Overall, evaluation of treatment response is potentially the most important clinical application of Fluorodeoxyglucose PET in TB, owing to its ability to distinguish active from inactive disease. Fluorodeoxyglucose PET and PET/CT may assist early diagnosis and facilitate differentiation between malignancies and TB, identification of extra pulmonary TB, staging of TB, and assessment of treatment response. The diagnosis and prognosis of residual disease is a bit of problem in spinal TB owing to artifacts produced by implants. PET scan proves to be important in this scenario. It also helps in measuring the effectiveness of treatment. It also recognizes the new lesions that may arise because of resistance to anti-tubercular drugs.
\end{abstract}

Keywords: Fluorodeoxyglucose; PET; CT; Tuberculosis

\section{Introduction}

Detection and localization of infectious and non-infectious foci in soft tissues is of primary importance for the management of patients with presumed or established inflammatory and septic diseases. X-rays, Ultrasound, CT, and MRI are effective in detecting inflammation whenever the lesion has caused changes in local anatomy, capillary permeability, or tissue water content [1]. When normal anatomic landmarks are lost or obscured, localization of inflammatory foci can best be accomplished with scintigraphic techniques. PET with 18FFDG is also useful to detect infection and inflammation foci [2]. High tissue radioactivity after administration of $18 \mathrm{~F}-\mathrm{FDG}$ corresponds to increased glucose uptake and consumption through the hexose monophosphate shunt, the main source of energy in chemo taxis and phagocytosis [3]. Activation of phagocytes, also known as respiratory burst activation, leads to increased 18F-FDG uptake [4]. In sterile inflammation, administered 18F-FDG is mainly taken up by neutrophils and macrophages [5]. High degree of 18F-FDG uptake is seen in neutrophils during acute phase of inflammation, while macrophages and polymorphonuclear leukocytes take up FDG during chronic phase. Like glucose, FDG is transported into cells by means of a glucose transporter protein. Once inside the cell, 18F-FDG is phosphorylated into 18F-FDG-6-phosphate. However, 18FFDG-6-phosphate cannot continue through glycolysis because it is not a substrate for glucose-6phosphate isomerase and is trapped within the cell. There are two major differences in mechanism of FDG uptake in tumor and inflammatory cells. Firstly, glucose-6-phosphatase levels are decreased in tumor cell, while it remains the same in inflammatory cells. This leads to washout of FDG from inflammatory cells. Secondly, there are much more increased Glut receptors in Tumor cells as compared to inflammatory cells. It has also been shown that FDG rapidly accumulates at the sites of bacterial infection and in reactive lymph nodes with a high target to-background ratio compared with other tracers, such as radiolabeled thymidine, L-methionine, gallium-67 citrate (67Ga-citrate), and iodine-125 human serum albumin (125I-HSA) [6,7] (Figure 1).

\section{Method}

A total of 10 patients who were operated for spinal tuberculosis were selected for the study. A PET scan was done at regular interval of follow up on $1^{\text {st }}$ month, $6^{\text {th }}$ month and 1 year. Upon study it revealed that the FDG uptake was reduced at the site of lesion in 9 patients and was completely absent by the end of 6 months. Upon Biopsy from the same site it was found to be Tuberculosis. The same patient was started on extended course of AKT. In 7 patients, the FDG uptake was absent at lesion site at even 1 year PET scan. However in 2 patients, it was revealed that there was uptake of FDG on PET scan, which suggested possibility of re-infection/noncompliance to treatment/resistant infection. Upon Biopsy from the same site it was found to be a multidrug resistant TB. These two patients were then started on MDR TB responsive treatment. A repeat PET scan after 6 months showed no more uptake of FDG which is suggestive of effective Koch's control.

\section{Discussion}

As 18 FFDG is known to accumulate in inflammatory cells such as neutrophils and activated macrophages at the site of inflammation this enables $18 \mathrm{~F}-\mathrm{FDG}-\mathrm{PET} / \mathrm{CT}$ to be the preferred modality for realtime assessment of disease activity over CXR, CT, and MRI. 18F-FDG PET/CT was superior in the identification of involved nodal basins compared to CT alone. This suggests that $18 \mathrm{~F}-\mathrm{FDG}$ PET is a valuable

*Corresponding author: Khandelwal RCS, Department of Orthopaedics, King Edward Memorial Hospital, Mumbai, India, Tel: +9892499162; E-mail: dhakejagdish9@gmail.com

Received October 24, 2018; Accepted November 16, 2018; Published November 21, 2018

Citation: Khandelwal RCS, Nathan S, Deo T, Dhake JM, Shetty NS (2018) Role of Fluorodeoxyglucose - Positron Emission Tomography Scan in Detection of Residual Pus Pocket Post-Operatively in Tuberculosis of Spine and Juxta Articular Tuberculosis. J Spine 7: 424. doi: 10.4172/2165-7939.1000424

Copyright: () 2018 Khandelwal RCS, et al. This is an open-access article distributed under the terms of the Creative Commons Attribution License, which permits unrestricted use, distribution, and reproduction in any medium, provided the original author and source are credited. 


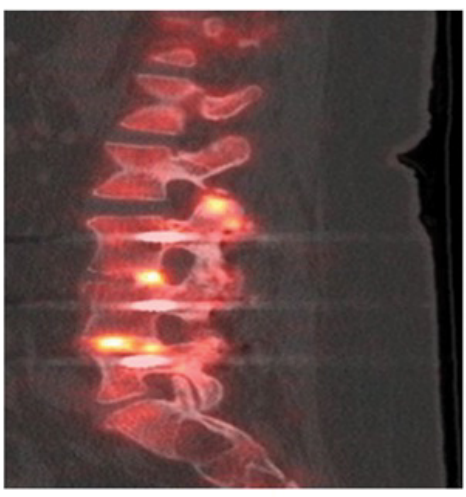

(a)

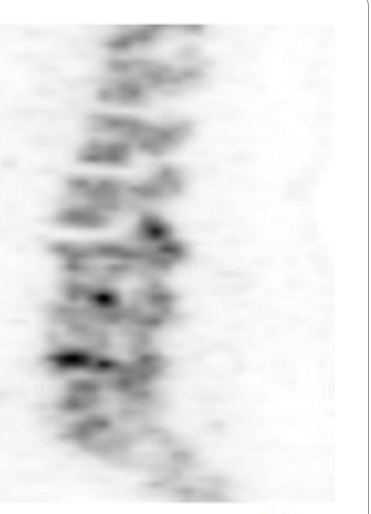

(b)
Figure 1: Tumor cells as compared to inflammatory cells.

imaging modality for the prediction of possible drug resistance thereby impacting on management. Although no imaging modality can confidently differentiate benign from malignant disease $18 \mathrm{~F}-\mathrm{FDG}$ $\mathrm{PET} / \mathrm{CT}$ has proven to be a valuable modality for the identification of the most appropriate biopsy site for histopathological evaluation and diagnosis. Secondly even though definitive diagnosis and exclusion of active TB infection by culture and examination of tissue specimens are always recommended, 18F-FDG-PET/CT may contribute to earlier detection/diagnosis. This suggests that 18F-FDG-PET/CT may be a useful tool in the assessment of the extent of active disease involvement prior to treatment and potentially for the assessment of treatment response.

\section{Conclusion}

$18 \mathrm{~F}-\mathrm{FDG}-\mathrm{PET} / \mathrm{CT}$ may be a useful tool in the assessment of the extent of active disease involvement prior to treatment and potentially for the assessment of treatment response. Preliminary work done on animal models as well as humans has demonstrated that quantitative 18F-FDG uptake can be used to monitor the effectiveness of treatment.

\section{References}

1. Heysell SK, Thomas TA, Sifri CD, Rehm PK, Houpt ER (2013) 18-fluorodeoxyglucose positron emission tomography for tuberculosis diagnosis and management: A case series. BMC Pulm Med 13: 14.

2. El-Haddad G, Zhuang H, Gupta N, Alavi A (2004) Evolving role of positron emission tomography in the management of patients with inflammatory and other benign disorders. Semin Nucl Med 34: 313-329.

3. Martinez V, Castilla-Lievre MA, Guillet-Caruba C, Grenier G, Fior R, et al. (2012) (18)F-FDG PET/CT in tuberculosis: an early non-invasive marker of therapeutic response. Int J Tuberc Lung Dis 16: 1180-1185.

4. Treglia G, Taralli S, Calcagni ML, Maggi F, Giordano A, et al. (2011) Is there a role for fluorine 18-fluorodeoxyglucose positron emission tomography and positron emission tomography/computed tomography in evaluating patients with mycobacteriosis? A systematic review. J Comput Assist Tomogr 35: 387-393.

5. Barry CE 3rd, Boshoff HI, Dartois V, Dick T, Ehrt S, et al. (2009) The spectrum of latent tuberculosis: rethinking the biology and intervention strategies. Nat Rev Microbiol 7: 845-555.

6. Lin PL, Flynn JL (2010) Understanding latent tuberculosis: A moving target. J Immunol 185: 15-22.

7. Ankrah AO, Van der Werf TS, De Vries EF, Dierckx RA, Sathekge MM, et al. (2016) PET/CT imaging of Mycobacterium tuberculosis infection. Clin Transl Imaging 4: 131-144. 